\title{
Sealed operation, and circulation and purification of gas in the HARPO TPC
}

\author{
M. Frotin ${ }^{1, a}$, P. Gros ${ }^{1, a}$, D. Attié 2 , D. Bernard ${ }^{1}$, V. Dauvois ${ }^{3}$, A. Delbart ${ }^{2}$, D. Durand ${ }^{3}$, Y. Geerebaert ${ }^{1}$, S. Legand ${ }^{3}$, \\ P. Magnier ${ }^{2}$, P. Poilleux ${ }^{1}$, and I. Semeniouk ${ }^{1}$ \\ ${ }^{1}$ LLR, Ecole Polytechnique, CNRS/IN2P3, 91128 Palaiseau, France \\ ${ }^{2}$ IRFU, CEA Saclay, 91191 Gif-sur-Yvette, France \\ ${ }^{3}$ DEN/DANS/DPC/SECR/LRMO, CEA Saclay, 91191 Gif-sur-Yvette, France
}

\begin{abstract}
HARPO is a time projection chamber (TPC) demonstrator of a gamma-ray telescope and polarimeter in the $\mathrm{MeV}-\mathrm{GeV}$ range, for a future space mission. We present the evolution of the TPC performance over a five month sealed-mode operation, by the analysis of cosmic-ray data, followed by the fast and complete recovery of the initial gas properties using a lightweight gas circulation and purification system.
\end{abstract}

\section{Introduction}

High-energy astronomy is hindered by the sensitivity gap between the energy ranges over which the Compton and the pair telescopes are efficient, making the $\gamma$-ray sky in the range $0.1-100 \mathrm{MeV}$ almost unexplored. Furthermore no polarimeter with a decent sensitivity above $1 \mathrm{MeV}$ has ever been flown in space.

On the low-energy side of the gap, a number of projects are aiming at an improved sensitivity with respect to the COMPTEL telescope [7].Of these, those using stacks of silicon wafers without any tungsten converter can also detect pair conversions [1-3] with an angular resolution of $\approx 1.5^{\circ} @ 100 \mathrm{MeV}$, a significant improvement over that of Fermi-LAT $\left(\approx 6^{\circ} @ 100 \mathrm{MeV}\right.$ [4]). These stacks can have some sensitivity to polarisation if the wafers are thin enough and provided that matching can be performed in the first wafer after the conversion wafer, i.e. that each of the hits in the $x$ direction can be assigned to one of the two hits in the orthogonal $(y)$ direction. In subsequent wafers, the azimuthal information carried by the pair is washed out by multiple scattering [5].

In the quest for even thinner wafers, it is tempting to use an even less dense detector such as a gas detector. With a spatial resolution of $100 \mu \mathrm{m}$, and with an optimal tracking in the presence of multiple scattering provided by a Kalman filter, an angular resolution of $\approx 0.4^{\circ} @ 100 \mathrm{MeV}$ can be achieved [6]. This provides a point-like source sensitivity as low as $10^{-6} \mathrm{MeV} /\left(\mathrm{cm}^{2} \mathrm{~s}\right) @ 100 \mathrm{MeV}$ with a $10 \mathrm{~kg}$ gas time projection chamber (TPC), while that of COMPTEL plateaued at $\approx 10^{-4} \mathrm{MeV} /\left(\mathrm{cm}^{2} \mathrm{~s}\right)$ [7]. In such gas detectors the dilution of the azimutal information carried by the pair is close to unity, so that the sensitivity to polarisation is excellent [8]. Finally track matching can easily be performed by comparing the distribution of the energy deposited along the tracks in the $x$ and $y$ directions

\footnotetext{
a corresp. authors: frotin@1lr.in2p3.fr, philippe.gros@1lr.in2p3.fr
}

thanks to the large fluctuation in the local deposition of energy along the track [9].

These unique features come at the cost of the need to maintain a good-quality gas in which the collection of the ionizing electrons is not affected by impurities with a large electron attachment. For example the EGRET gas $(99.5 \%$ neon, $0.25 \%$ argon, and $0.25 \%$ ethane) became contaminated by the breakdown of ethane by spark chamber firings and to a small degree by residual outgassing. The five planned gas exchanges were carried out once per year, after which the lack of fresh gas became a serious issue [10]. These concerns have triggered the development of detectors built out of low outgassing rate materials. For example, the design of the GEMS X-ray polarimeter TPC involves gas electron multipliers (GEM) made of copper clad liquid crystal polymer (LCP), and a dielectric structure of polyetheretherkeytone (PEEK) on a gold-plated titanium frame. This gives it an expected lifetime of 23 year [11].

We report here on a gas purity study performed with the HARPO detector, a TPC prototype of a $\gamma \rightarrow e^{+} e^{-}$telescope built with techniques much better suited to our actual budget and that we have studied in the laboratory with radioactive sources [12] and with cosmic rays [13], and that we have tested recently [14] in a $\gamma$-ray beam.

\section{Description of the HARPO TPC}

HARPO is a $30 \mathrm{~cm}$ cubic TPC surrounded by 6 scintillator plates, each equipped with two wavelength shifter bars and two photomultipliers (PMTs) for trigger and background rejection. The gas container is an aluminum cylinder designed to be operated from few mbar up to 5 bar in sealed mode (Fig. 1). With a mixture of $95 \%$ of argon 5.0 and $5 \%$ of $\mathrm{iC}_{4} \mathrm{H}_{10} 3.5$ and at a $220 \mathrm{~V} / \mathrm{cm}$ drift electric field, the electron drift velocity is about $3 \mathrm{~cm} / \mu \mathrm{s}$. The TPC endplate is a "hybrid" MicroPattern Gas Detector (MPGD) 
composed of two GEM foils[15] with $2 \mathrm{~mm}$ spacing above a $128 \mu \mathrm{m}$ amplification gap bulk-micromegas[16]. The anode collection plane is segmented in $2 \times 288$ strips in the two orthogonal $x, y$ directions and the signal is read by an electronic chain composed of two T2K/TPC Front-End cards[17], two FEMINOS back-end cards and a Trigger Clock Module [18]. A simple coincidence between scintillators is used here for triggering on cosmic rays.

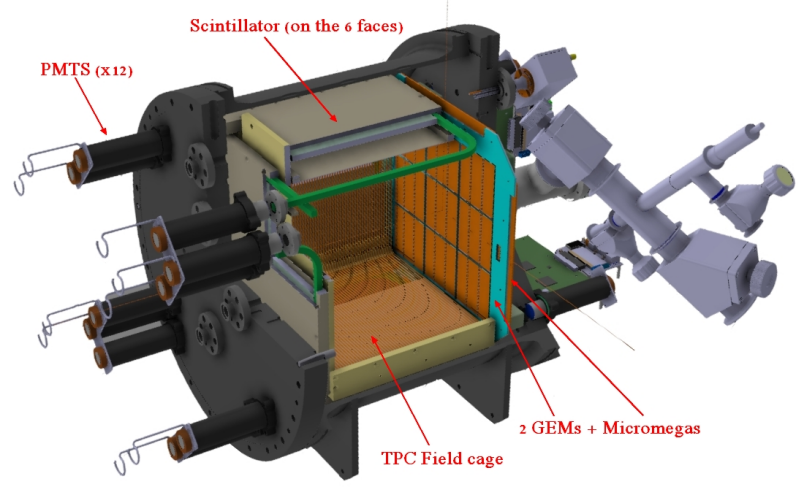

Figure 1. A 3D CAD drawing of the HARPO demonstrator.

\section{Description of the circulation and purification system (CAPS)}

We used a circulator composed of a simple turbine (Fig. 2, left). Full schematics of the circulation system is shown in Fig. 3 The gas was lead through an Oxisorb purification cartridge that can absorb up to $0.5 \mathrm{~L} \mathrm{H}_{2} \mathrm{O}$ and $0.1 \mathrm{~L} \mathrm{O}_{2}$, with output purity as low as $<5 \mathrm{ppb}$ for $\mathrm{O}_{2}$ and $30 \mathrm{ppb}$ for $\mathrm{H}_{2} \mathrm{O}$ [19]. We measured the gas flow with a F-111B Bronkhorst mass flowmeter (Fig. 3, I10) that was calibrated for our gas mix at 2 bar and $20{ }^{\circ} \mathrm{C}$. We monitored the gas pressure and temperature with a Keller EV-120/PA(A)-33X sensor (Fig. 3, I4 and I5) with ranges of $0-50{ }^{\circ} \mathrm{C}$ and $0-10$ bar and resolutions of $0.01^{\circ} \mathrm{C}$ and $1 \mathrm{mbar}$. The outgassing areas inside the vessel amount to $0.82 \mathrm{~m}^{2}$ Aluminum, $3.50 \mathrm{~m}^{2} \mathrm{PVC}$, $0.92 \mathrm{~m}^{2} \mathrm{PCB}, 1.63 \mathrm{~m}^{2}$ PVT, for a gas volume $V_{\mathrm{det}}=84 \mathrm{~L}$.

We tested the gas circulation system with a helium leak detector (ASM 310) down to $3.3 \cdot 10^{-8} \mathrm{mbar} \cdot \mathrm{L} / \mathrm{s}$, after which we opened the connection between the CAPS and the detector. This caused a pressure drop from $2015 \mathrm{mbar}$ to 1960 mbar at $27^{\circ} \mathrm{C}$, with a flow of $1.5 \mathrm{~L} / \mathrm{h}$. On July 20 2015, we opened the Oxisorb purificator in vacuum which lead to a new pressure drop to 1932 mbar when the connection to the detector was opened. The CAPS then ran continuously, with a flow through the filter of $f_{\text {circ }}=1.4 \mathrm{~L} / \mathrm{h}$. In these conditions we expect the water and oxygen content in the gas, $C$, to evolve following a law of the form:

$$
C=C_{0} e^{-t / \tau_{\text {purif }}}+K_{\text {cont }} \tau_{\text {purif }},
$$

with $\tau_{\text {purif }}=V_{\text {det }} / \epsilon_{\text {purif }} f_{\text {circ }}$, where $C_{0}$ is the original content and $K_{\text {cont }}$ is the contamination rate from leaks and outgassing. In case the purification efficiency of the filter is $\epsilon_{\text {purif }}=1$, the purification rate is equal to $\tau_{\text {purif }}=60 \mathrm{~h}$.

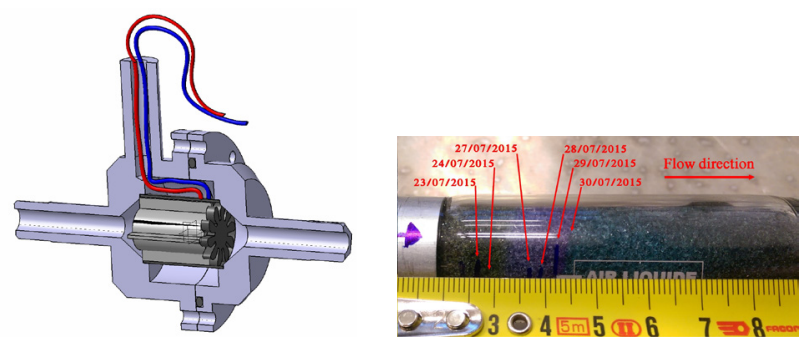

Figure 2. (Left) Sectional view of the circulator (CEA patent BD15730SG). (Right) Picture of the Oxisorb cartridge. The progression of the purple-coloured region over 10 days of continuous use is indicated.

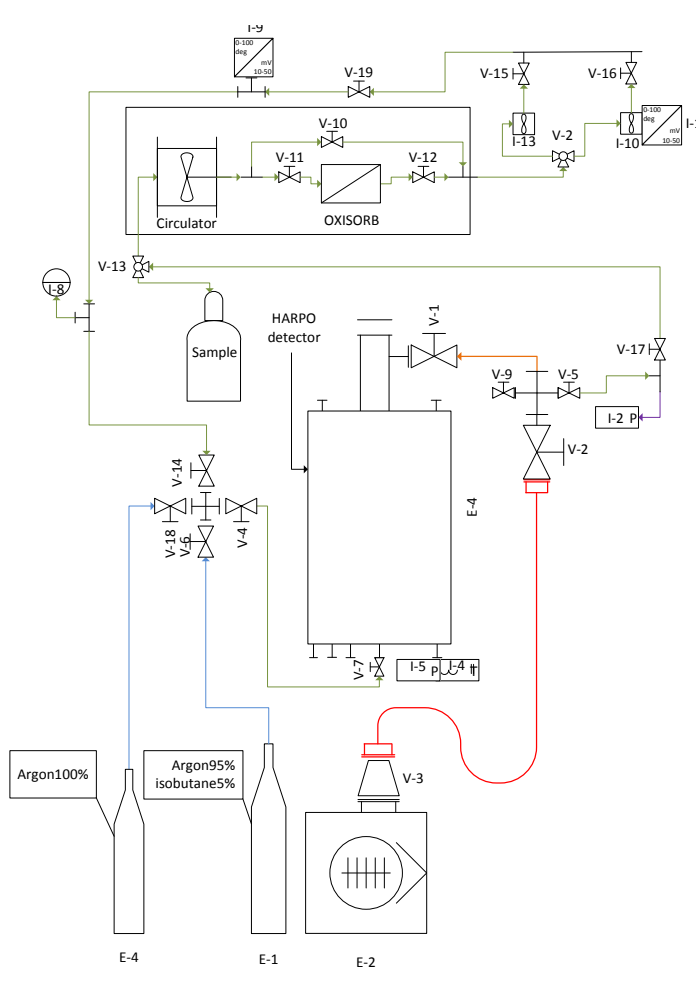

Figure 3. Layout of the gas system.

The Oxisorb purificator color ranges from blue to dark brown with the quantity of $\mathrm{H}_{2} \mathrm{O}$ and $\mathrm{O}_{2}$ absorbed. At the beginning of the purification, we observed a purple ring of $3 \mathrm{~mm}$ thickness growing $3 \mathrm{~mm}$ per day during the first 4 days. It stabilised at $12 \mathrm{~mm}$ after a few days (Fig. 2). This indicates that the cartridge was never saturated.

Several gas samples were taken for analysis, which lead to density drops (lines 1, 2, and 3 on Fig. 7). Before and after every operation that affected the gas in the vessel, data was taken with the TPC for consistency checks.

\section{Data taking and analysis}

We took cosmic-ray data once per week from February to July, with the detector set so that the drift field was vertical. Most runs were one hour long, recording a few 10,000 events. After starting the purification on July 20 
we took data more frequently. The high voltage was only turned on when taking data. Due to the vertical detector orientation, most of the recorded tracks are approximately aligned with the drift direction. Figure 4 shows the $X, t$ and $Y, t$ projections for a typical event. We select events with

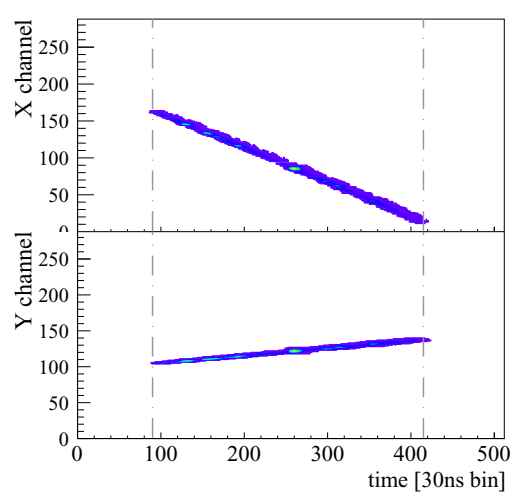

Figure 4. A cosmic-ray event in HARPO. The "maps" show the charge distributions in the projection X-time and Y-time.

a single track in each projection, with a high momentum muon selection based on the $\chi^{2}$ cut of a linear fit. For each time bin $t_{i}(30 \mathrm{~ns} \approx 1 \mathrm{~mm})$, we obtain the charge deposited by a track, $Q\left(t_{i}\right)$, summing over all the channels associated with the track. After an angle-effect correction using the direction $\vec{u}$ of the track,

$$
\frac{d E}{d x}\left(t_{i}\right)=Q\left(t_{i}\right) \times u_{z},
$$

we obtain the $d E / d x\left(t_{i}\right)$ in ADC units, that is up to an overall factor that includes the gain of the amplification of both the MPGDs and that of the readout electronics. The result-

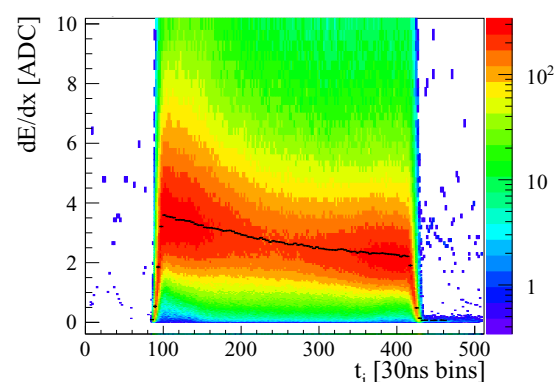

Figure 5. Normalised charge as a function of the drift time for a 6000 s cosmic-ray run.

ing distribution (Fig. 5) is also affected by threshold and saturation effects. To mitigate them, we estimate the most probable value (MPV, solid line in Fig. 5) of the charge for each time bin with a Landau-function fit. From this distribution we extract :

The drift velocity. As the tracks are selected to traverse the full TPC length, the drift-time duration provides a measurement of the drift velocity.

The gain. The height of the distribution at short drift times is proportional to the amplification gain.
Table 1. Gas content from mass spectrometry (volume/volume). Note that the set-up was not sensitive to $\mathrm{H}_{2} \mathrm{O}$.

\begin{tabular}{|l|c|c|c|c|}
\hline \multicolumn{2}{|c|}{ Sample \# } & $\begin{array}{c}1 \\
\text { Compound }\end{array}$ & $\begin{array}{c}2 \\
\text { July 08 }\end{array}$ & $\begin{array}{c}3 \\
\text { Aug. 27 }\end{array}$ \\
Sept. 17 \\
\hline $\mathrm{iC}_{4} \mathrm{H}_{10}$ & $\%$ & 5.10 & 4.42 & 4.49 \\
$\mathrm{O}_{2}$ & ppm & 180 & $<20$ & $<20$ \\
$\mathrm{CO}$ & ppm & 190 & 250 & 130 \\
$\mathrm{CO}_{2}$ & ppm & 120 & 160 & 130 \\
$\mathrm{~N}_{2}$ & ppm & 620 & 890 & 850 \\
\hline
\end{tabular}

The attenuation. The signal loss along the drift from electron capture is visible in Fig. 5. We fit the central part of the distribution, which is less affected by the threshold and saturation effects, with an exponential function.

These measurements are affected by systematic effects. Since the detector configuration was kept unchanged throughout the data taking, they cancel in the relative value compared to a reference run, chosen to be run 2008 (Feb. 18th), i.e. right after the fresh gas fill. Figure 6 shows the relative charge distribution as a function of the drift distance for several runs taken at a few weeks interval.

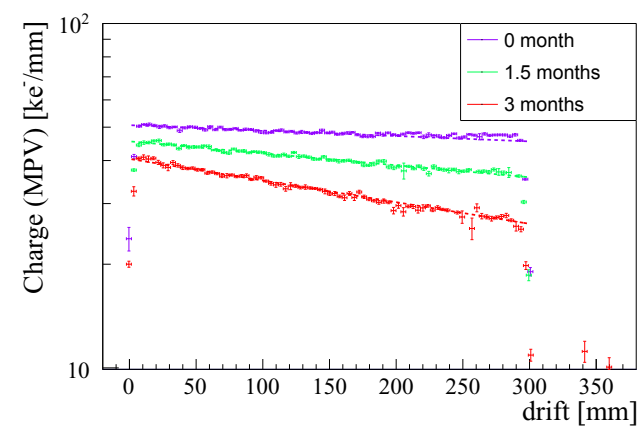

Figure 6. Charge as a function of the drift distance, for three runs, 6 weeks apart, normalised to the reference run 2008.

\section{Results}

For the first five months, while the TPC was sealed, we observe a steady degradation of the gain, of the drift velocity and of the electron attenuation (Fig. 7). After we turned the CAPS on, the drift velocity and the attenuation got back to their initial values. We corrected for the gain changes due to the density decrease induced by our extraction of gas samples and extension of the volume with the CAPS circuit, by adjusting the MPGD voltages. The pressure change also affected the drift velocity but to a negligible extent as shown by Garfield simulations[20], compared to the observed effect.

Gas samples were collected before (\#1, line 3 on Fig.7), and respectively five weeks (\#2) and eight weeks (\#3) after activating the purification system. They were analysed in a high resolution $(R=2800)$ direct-injection THERMO MAT 271 mass spectrometer (Fig. 8, Table 1).

The relative amount of $\mathrm{O}_{2}$ and $\mathrm{N}_{2}$ before purification is consistent with that of air, due to either leaks or outgassing. Contaminations by $\mathrm{CO}$ and $\mathrm{CO}_{2}$ probably originate from outgassing from plastic elements. 


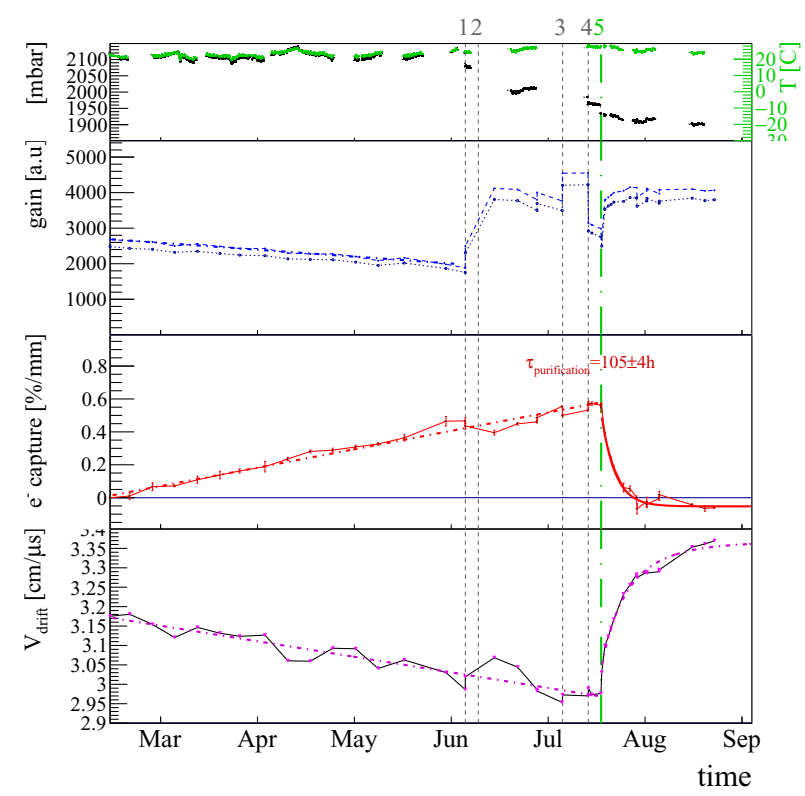

Figure 7. Time evolution of the gain, of the attenuation and of the drift velocity. We can see several variations of the gain due to the pressure loss when gas samples were extracted. For the first five months during which the TPC was sealed we see a regular degradation of all three parameters. On July 20 (line 5), the CAPS was turned on.

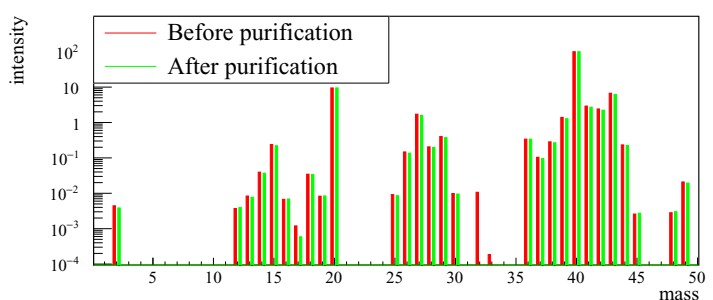

Figure 8. Mass spectroscopy results for samples taken before and after purification. The overall composition is similar, except for $\mathrm{N}=32\left(\mathrm{O}_{2}\right)$, that disappeared in the second sample.

After purification, the residual oxygen content is found to be lower than the detection threshold of the spectrometer. We also note a small but significant decrease of the amount of isobutane content upon purification after which it stabilized, an effect that is not understood.

\section{Discussion}

Garfield++ simulations [20] show that the degradation of the attenuation during sealed operation, attributed to electron attachment, is proportional to the oxygen content. After purification was started the effect disappears exponentially with a characteristic time $\tau_{\text {purif }}=105 \pm 4 \mathrm{~h}$ (Fig. 7). The observed time constant is larger than the computed value, most likely due to the limitation of gas circulation inside the almost closed field cage. We also observe a decrease of the drift velocity, which cannot be associated with the oxygen contamination and which is recovered after purification, and therefore that can be attributed to water contamination.

After purification, the drift velocity was measured to be $6.0 \pm 0.5 \%$ higher than its initial value. From the Garfield simulation, this can be explained by the cumulative effects of the reduced gas pressure from 2.1 to 1.9 bar $(+4 \%)$ and of the measured decrease of the isobutane content from $5.1 \%$ to $4.5 \%(+3 \%)$.

\section{Conclusion}

We have successfully operated a sealed TPC over five months, with a clear but manageable gas degradation. We used the data from cosmic rays in the TPC to monitor the relevant parameters of the gas (drift velocity, gain, attenuation). We then purified the gas with a simple, low-power circulation system and observed a fast and complete recovery of the initial parameters. If operated routinely, for example on a space mission, the system should therefore enable a stable performance for several years.

This work is funded by the French National Research Agency (ANR-13-BS05-0002)

\section{References}

[1] "ASTROGAM", proposal submitted for the ESA M4 Mission Programme, Jan. 2015, http://astrogam.iaps.inaf.it/

[2] A. A. Moiseev et al., arXiv:1508.07349 [astro-ph.IM].

[3] X. Wu et al., Proc. SPIE 9144, 91440F (2014) [arXiv:1407.0710 [astro-ph.IM]].

[4] M. Ackermann et al. [Fermi-LAT Collaboration], Astrophys. J. Suppl. 203 (2012) 4 [arXiv:1206.1896 [astro-ph.IM]].

[5] D. Bernard, The gamma-ray sky with astrogam, Second ASTROGAM Workshop, March 2015, Paris.

[6] D. Bernard, Nucl. Instrum. Meth. A 701, 225 (2013) [Erratumibid. A 713, 76 (2013)] [arXiv:1211.1534 [astro-ph.IM]].

[7] V. Schönfelder, New Astr. Rev. 48 (2004) 193.

[8] D. Bernard, Nucl. Instrum. Meth. A 729 (2013) 765, [arXiv:1307.3892 [astro-ph.IM]].

[9] D. Bernard, 12th Pisa Meeting on Advanced Detectors, Isola d'Elba, Italy, May 2012, Nucl. Instrum. Meth. A 718, 395 (2013), [arXiv:1210.4399 [astro-ph.IM]],

[10] J. A. Esposito et al., Astrophys. J. Suppl. 123 (1999) 203.

[11] J. E. Hill et al., Proc. SPIE 8859, San Diego, United States, Aug. 2013, 885900 .

[12] P. Gros et al., TIPP14, 2-6 June 2014 Amsterdam, proceedings PoS(TIPP2014)133

[13] D. Bernard et al., SPIE 9144, June 2014, Montréal, Canada, 91441M; arXiv:1406.4830 [astro-ph.IM].

[14] S. Wang et al., TPC2014, Dec. 2014 Paris, J.Phys.Conf.Ser. 650 (2015) 012016, arXiv:1503.03772 [astro-ph.IM].

[15] F. Sauli, Nucl. Instrum. Meth. A 386, (1997) 531.

[16] I. Giomataris et al., Nucl. Instrum. Meth. A 560, (2006) 405.

[17] P. Baron et al., IEEE Trans. Nucl. Sci. 55 (2008) 1744.

[18] D. Calvet, IEEE Trans. Nucl. Sci. 61 (2014) 675.

[19] Oxisorb, air liquide, ref 123342.

[20] R. Veenhof et al., http://garfieldpp.web.cern.ch/garfieldpp/ 